\title{
Mecanismos de hipo e hiper alfalipoproteinemia en individuos adultos chilenos
}

\author{
Ada Cuevas $\mathrm{M}^{1}$, Verónica Alvarez $\mathrm{V}^{\mathbf{1} a}$, Ana María Acosta $\mathrm{B}^{1}$, \\ Marcela Altayó $\mathrm{F}^{2}$, Joaquín Montero $\mathrm{L}^{3}$, Attilio Rigotti $\mathrm{R}^{2}$. \\ Mechanisms of hypo and \\ hyper alphalipoproteinemia \\ in Chilean adults
}

\begin{abstract}
Background: High density lipoprotein (HDL) cholesterol is inversely associated to atherosclerotic cardiovascular risk. Disturbances in HDL cholesterol plasma leves are frecuent in the Chilean population, however the pathophysiological mechanisms are unknown. Aim: To evaluate the mechanisms involved in the hypo and hyper alfalipoproteinemias in Chilean subjects. Materials and Methods: Twenty three subjects with hyperalphalipoproteinemia and 12 with hypoalphalipoproteinemia, paired with control subjects (colHDL between 35 and $55 \mathrm{mg} / \mathrm{dl}$ ) were studied. We measured plasma lipids, subfractions and sizing of HDL particles and enzymatic activity of cholesteryl ester transfer protein (CETP), lecithin: cholesterol acyltransferase (LCAT), lipoprotein lipase (LPL) and hepatic lipase (LH). Results: Subjects with hyperalphalipoproteinemia showed significantly higher levels of total HDL-cholesterol ( $70 \pm 2$ vs $44 \pm 1 \mathrm{mg} / \mathrm{dl}$ ), HDL 2 ( $30 \pm 3$ vs $5 \pm 1 \mathrm{mg} / \mathrm{dl}$ ), Apo A I ( $175 \pm 3$ vs $146 \pm 4 \mathrm{mg} / \mathrm{dl}$ ), lower HL activity $(23,7 \pm 0,8$ vs $32,4 \pm 1,8 \mathrm{mmol} / \mathrm{h} / \mathrm{l})$ and HDL particles of greater size, compared to their controls. Subjects with hypoalphalipoproteinemia, showed significantly lower levels of total HDL-cholesterol ( $26 \pm 1$ vs $48 \pm 2 \mathrm{mg} / \mathrm{dl}$ ), HDL 3 ( $21 \pm 1$ vs $40 \pm 2 \mathrm{mg} / \mathrm{dl})$, Apo A I ( $107 \pm 5$ vs $145 \pm 7$ $\mathrm{mg} / \mathrm{dl})$, lower LCAT activity $(18,6 \pm 1,9 \mathrm{vs} 26,2 \pm 1,6 \mathrm{nmol} / \mathrm{h} / \mathrm{ml})$ and smaller HDL particles, compared to their controls. Conclusion: Changes in hepatic lipase and lecithin cholesterol acyltransferase activities may explain the hyper and hypo alphalipoproteinemia respectively, in Chilean subjects (Rev Méd Chile 2004; 132: 421-8).
\end{abstract}

(Key Words: Lecithin acyltransferasa deficiency; Lipoproteins, HDL; Lipoproteins, HDL cholesterol; Lipoproteins, VLDL cholesterol)

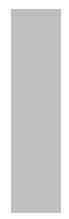

\begin{abstract}
Recibido el 31 de marzo, 2003. Aceptado en versión corregida el 23 diciembre, 2003.
Trabajo financiado por la Beca de Investigación Clínica Sociedad Médica de Santiago-Laboratorio Saval 2000 y el Proyecto FONDECYT \#8990006.

1Departamentos de Nutrición, Diabetes y Metabolismo, ${ }^{2}$ Gastroenterología y ${ }^{3}$ Medicina Interna, Facultad de Medicina, Pontificia Universidad Católica de Chile. Santiago, Chile. aTesista del Programa de Magíster en Nutrición, Pontificia Universidad Católica de Chile.
\end{abstract}

Correspondencia a: Dra. Ada Cuevas Marín. Departamento de Nutrición, Diabetes y Metabolismo, Facultad de Medicina, Pontificia Universidad Católica. Marcoleta 367, Santiago. Fono: (562) 6863862. Fax: (562) 6338298. E mail: acuevas@rdc.cl 
$\mathrm{D}$ iversos estudios epidemiológicos han establecido que un bajo nivel plasmático de colesterol-HDL constituye un factor de riesgo independiente de enfermedad cardiovascular ateroesclerótica ${ }^{1,2}$. Inversamente, un colesterol-HDL plasmático elevado constituye un factor protector frente al desarrollo de enfermedades cardiovasculares. Incluso, un estudio de intervención con gemfibrozilo en pacientes portadores de enfermedad coronaria e hipoalfalipoproteinemia demostró una reducción de eventos cardiovasculares asociado al incremento de colesterol-HDL ${ }^{3}$. Los mecanismos por los cuales el colesterol-HDL disminuye el riesgo de ateroesclerosis son variados e incluyen el transporte reverso de colesterol, la disminución de la oxidación de las LDL, el posible efecto antiinflamatorio, la regulación de factores de coagulación y la mejoría de la función endotelial ${ }^{4}$.

Los niveles plasmáticos de colesterol HDL se encuentran determinados por una compleja interacción de factores ambientales, metabólicos y genéticos. Dentro de los factores secundarios que tienen impacto en el colesterol-HDL destacan el ejercicio, tabaquismo, cantidad y calidad de ingesta grasa y el consumo de alcohol. Por otro lado, los factores metabólicos y genéticos que afectan el colesterol-HDL plasmático son las hipertrigliceridemias primarias, diabetes mellitus, obesidad y variantes de los genes que codifican elementos claves del metabolismo de HDL, tales como la apolipoproteína A-I (apo A-I), principal constituyente proteico de las HDL, las lipasas hepática y lipoproteica, la enzima de transferencia de ésteres de colesterol (CETP), la enzima esterificadora de colesterol libre (LCAT), el transportador ABCA1 y el receptor de HDL SR-BI ${ }^{5-7}$. La comprensión detallada de los mecanismos involucrados en las dislipidemias del colesterol-HDL debiera permitir un manejo más racional de esta importante variable metabólica determinante del riesgo cardiovascular ateroesclerótico.

En Chile, alrededor de 10\% de las mujeres y $20 \%$ de los hombres adultos presentan bajos niveles sanguíneos de colesterol $\mathrm{HDL}^{8}$. Sin embargo, y a pesar de la importancia de las enfermedades cardiovasculares en nuestra población, no se han realizado estudios que establezcan los mecanismos determinantes de hipo e hiper alfalipoproteinemias en sujetos adultos chilenos.

\section{MATERIALES Y MÉTODOS}

Se realizó un estudio caso-control que incluyó 12 individuos adultos portadores de hipoalfalipoproteinemia (colesterol-HDL $\leq 30 \mathrm{mg} \%$ ) y 23 con hiperalfalipoproteinemia (colesterol-HDL $\geq 60$ $\mathrm{mg} \%)$, los cuales fueron reclutados del policlínico de dislipidemias del Departamento de Nutrición, Diabetes y Metabolismo y de las bases computacionales de pacientes que acuden a chequeos médicos en la Facultad de Medicina de la Pontificia Universidad Católica de Chile. Cada sujeto fue pareado por sexo, edad e índice de masa corporal (IMC) con un sujeto control con colesterol-HDL entre 35 y $55 \mathrm{mg} / \mathrm{dl}$. Se excluyeron del estudio sujetos portadores de hipertrigliceridemia (triglicéridos $>250 \mathrm{mg} / \mathrm{dl}$ ), tabaquismo, enfermedades hepáticas o renales, hipotiroidismo, diabetes mellitus, enfermedad coronaria clínica, alcoholismo, uso de estrógenos, terapia hipolipemiante, alteraciones de la coagulación y enfermedades inflamatorias recientes. Todos los sujetos dieron su consentimiento informado para participar en el estudio y el protocolo fue aprobado por el Comité de Etica de la Facultad de Medicina de la Pontificia Universidad Católica de Chile.

Posterior a $12 \mathrm{~h}$ de ayuno, los pacientes se sometieron a extracción de una muestra de sangre venosa para realizar las determinaciones de lípidos plasmáticos, subfracciones de HDL, Apo A-I, tamaño de HDL, actividad de LCAT y CETP. Además, se tomó una segunda muestra de sangre 15 min después de una inyección endovenosa de heparina (70 unidades por kilo de peso) para la determinación de lipasa hepática (LH) y lipoproteica (LPL) en plasma.

Los niveles de colesterol y triglicéridos totales se determinaron por métodos enzimáticos. El colesterol-HDL se midió después de la precipitación de las lipoproteínas plasmáticas no HDL con ácido fosfotúngstico. El colesterol-LDL fue calculado mediante fórmula de Friedewald. Las subfracciones de HDL (HDL2 y HDL3) fueron determinadas por precipitación con dextran sulfato ${ }^{9}$. Los niveles de Apo A-I plasmática fueron medidos por un método inmunoturbidimétrico. El tamaño de las partículas de HDL se analizó por cromatografía de filtración de alta resolución (FPLC).

Las actividades de las enzimas lipasa hepática y lipasa lipoproteica fueron determinadas 
por el método de Nielson-Ehle y Ekman ${ }^{10}$. La actividad de CEPT fue evaluada por un método radioactivo que mide la transferencia de colesterol esterificado marcado con tritio entre partículas de HDL y LDL (Amersham Pharmacia Biotech, Uppsala, Suecia). La actividad enzimática de LCAT fue determinada por el método de Manabe et $\mathrm{al}^{11}$.

Estadística. El análisis estadístico de los resultados se realizó mediante software computacional. Para analizar la significancia estadística de las diferencias entre los diferentes grupos estudiados, se utilizó el test de Student, test de varianza y $\chi^{2}$. Para evaluar las correlaciones entre las distintas variables medidas, se aplicó el método de análisis de regresión simple 0 múltiple. Un valor de $p<0,05$ fue considerado como un índice de diferencia estadísticamente significativa.

\section{RESULTADOS}

Características generales. El grupo con hiperalfalipoproteinemia incluyó 20 mujeres y 3 hombres con un promedio de edad de 50 $\pm 4,3$ años e IMC de $25 \pm 1,0$ $\mathrm{kg} / \mathrm{mt}^{2}$, sin diferencias estadísticamente significativas con sus respectivos controles. El grupo con hipoalfalipoproteinemia incluyó 4 mujeres y 8 hombres con un promedio de edad de 45 $\pm 3,9$ años e IMC de $27 \pm 1,0 \mathrm{~kg} / \mathrm{mt}^{2}$, siendo ambos parámetros estadísticamente similares a su grupo control (Tabla 1). Todos los sujetos cumplían los criterios de inclusión y exclusión antes mencionados. También mantenían una actividad física liviana y ninguno de ellos (casos y controles) realizaba algún tipo de alimentación especial o restricción dietaria.

Lípidos plasmáticos (Tabla 2). Como era esperable por los criterios de selección, los sujetos portado-

Tabla 1. Características generales de los individuos con hiper e hipo alfalipoproteinemia y sus respectivos controles

\begin{tabular}{|lcccc|}
\hline Característica & \multicolumn{2}{c}{ Hiperalfalipoproteinemia } & \multicolumn{2}{c|}{ Hipoalfalipoproteinemia } \\
& $\begin{array}{c}\text { Casos } \\
(\mathrm{n}=23)\end{array}$ & $\begin{array}{c}\text { Controles } \\
(\mathrm{n}=23)\end{array}$ & \multicolumn{2}{c|}{ Casos } \\
$(\mathrm{n}=12)$ & $\begin{array}{c}\text { Controles } \\
(\mathrm{n}=12)\end{array}$ \\
\hline Sexo (M:H) & $20: 3$ & $20: 3$ & $4: 8$ & $4: 8$ \\
Edad (años) & $50,0 \pm 4,3$ & $48,0 \pm 3,2$ & $45,0 \pm 3,9$ & $44,0 \pm 4,0$ \\
IMC (kg/mt $\left.{ }^{2}\right)$ & $25,0 \pm 1,0$ & $26,0 \pm 1,0$ & $27,0 \pm 1,0$ & $28,0 \pm 1,0$ \\
\hline
\end{tabular}

$\mathrm{H}=$ hombres, $\mathrm{M}=$ mujeres. $\mathrm{IMC}=$ Indice de masa corporal.

Valores son expresados como promedio \pm error estándar (ES).

Tabla 2. Niveles de lípidos y Apo Al plasmáticos en sujetos con hipo e hiper alfalipoproteinemia

\begin{tabular}{|lcccc|}
\hline $\begin{array}{l}\text { Lípidos } \\
(\mathrm{mg} / \mathrm{dl})\end{array}$ & \multicolumn{2}{c}{$\begin{array}{c}\text { Hiperalfalipoproteinemia } \\
\text { Casos } \\
(\mathrm{n}=23)\end{array}$} & $\begin{array}{c}\text { Controles } \\
(\mathrm{n}=23)\end{array}$ & \multicolumn{2}{c|}{$\begin{array}{c}\text { Hipoalfalipoproteinemia } \\
\text { Casos }\end{array}$} & $\begin{array}{c}\text { Controles } \\
(\mathrm{n}=12)\end{array}$ & \\
& $208 \pm 10$ & $194 \pm 11$ & $185 \pm 10$ & $188 \pm 9$ \\
Colesterol total & $99 \pm 10$ & $114 \pm 12$ & $134 \pm 18$ & $98 \pm 9$ \\
Triglicéridos & $118 \pm 8$ & $128 \pm 10$ & $132 \pm 8$ & $120 \pm 9$ \\
Colesterol LDL & $70 \pm 2$ & $44 \pm 1^{*}$ & $26 \pm 1$ & $48 \pm 2^{* *}$ \\
Colesterol HDL & $30 \pm 3$ & $5 \pm 1^{*}$ & $5 \pm 1$ & $8 \pm 2$ \\
HDL 2 & $40 \pm 3$ & $38 \pm 1$ & $21 \pm 1$ & $40 \pm 2^{* *}$ \\
HDL 3 & $6,7 \pm 0,9$ & $3,2 \pm 0,5^{*}$ & $3,3 \pm 0,5$ & $5,1 \pm 0,9$ \\
Triglicéridos de HDL & $175 \pm 3$ & $146 \pm 4^{*}$ & $107 \pm 5$ & $145 \pm 7^{* *}$ \\
Apo AI & & & & \\
\hline
\end{tabular}

Valores son expresados como promedio \pm ES.

${ }^{*} p<0,05$ versus hiperalfalipoproteinemia. ${ }^{*} p<0,05$ hipoalfalipoproteinemia. 


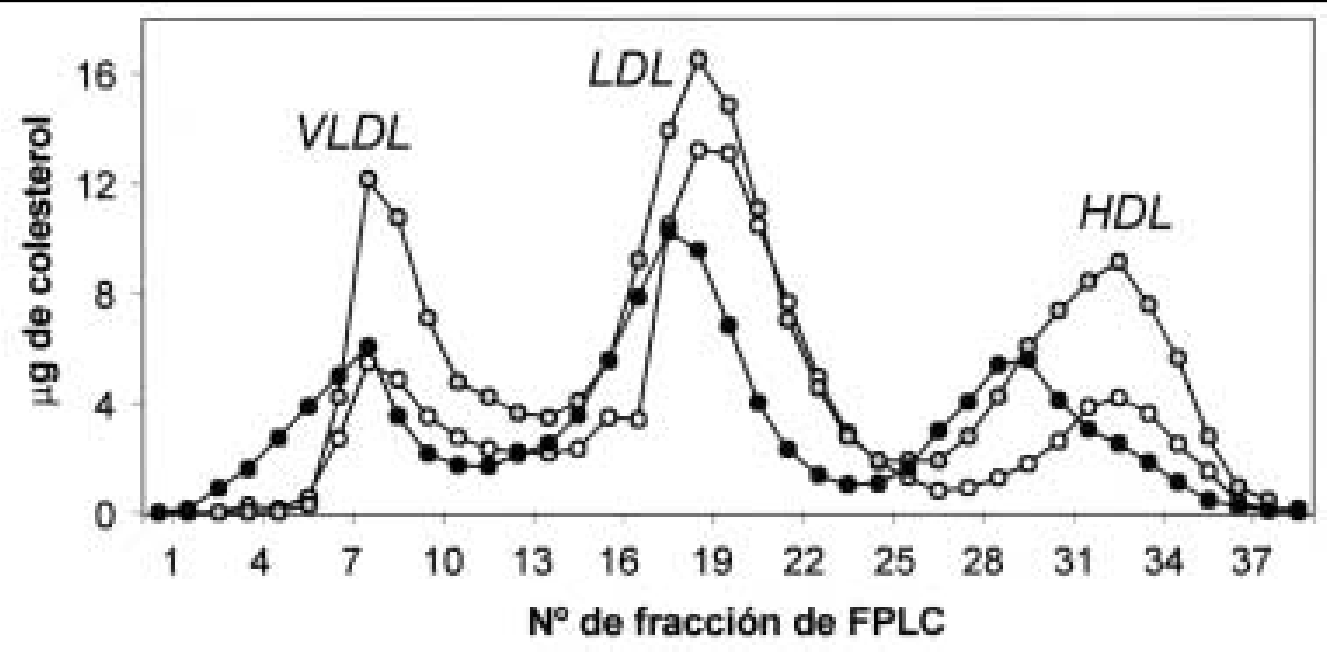

FIgURA 1. Tamaño de las partículas de HDL por cromatografía de filtración de alta resolución (FPLC). Círculos blancos = control; círculos grises $=$ hiperalfalipoproteinemia con HDL de tamaño normal; círculos negros = hiperalfalipoproteinemia con HDL de tamaño grande.

res de hiperalfalipoproteinemia e hipoalfalipoproteinemia presentaron niveles de colesterol-HDL significativamente más altos y más bajos en comparación a su grupo control. Las concentraciones plasmáticas de colesterol total, triglicéridos y colesterol-LDL fueron similares en los hiper e hipo alfalipoproteinémicos respecto a sus correspondientes controles.

Subfracciones de HDL (Tabla 2). Los sujetos con hiperalfalipoproteinemia presentaron niveles significativamente más altos de colesterol HDL2 en comparación a sus controles. El 57\% del total del colesterol-HDL correspondió a HDL3 y 43\% a HDL2. En cambio, en su respectivo grupo control, 80\% del total de HDL correspondió a HDL3 y sólo $20 \%$ a HDL2. En el grupo con hipoalfalipoproteinemia los niveles de HDL3 fueron significativamente más bajos en comparación a sus controles, correspondiendo esta subfracción a $81 \%$ del total de HDL y 19\% a HDL2, similar a lo detectado en sus controles, los cuales presentaron porcentajes de 83 y 17\% respectivamente.

Triglicéridos en HDL (Tabla 2). Los sujetos con hiperalfalipoproteinemia presentaron un mayor contenido de triglicéridos en HDL en comparación a su grupo control. No se detectaron diferencias en los individuos con hipoalfalipoproteinemia en comparación a sus respectivos controles.

Apo A-I (Tabla 2). Los niveles plasmáticos de Apo AI fueron significativamente más altos en el grupo de hiperalfalipoproteinemia en comparación a su grupo control. En cambio, en los individuos con hipoalfalipoproteinemia los niveles de Apo AI fueron significativamente menores en relación al control.

Tamaño de partículas de HDL (Figura 1). El 32\% (7/22) de los sujetos con hiperalfalipoproteinemia presentaron HDL de mayor tamaño en comparación a su control y 68\% (15/22) presentó HDL de tamaño normal. En los casos de hipoalfalipoproteinemia, 22\% (2/9) presentó HDL de tamaño más pequeño y 78\% (7/9) HDL de tamaño semejante a su respectivo control.

Actividad de enzimas plasmáticas involucradas en el metabolismo de HDL (Tabla 3). Los individuos con hiperalfalipoproteinemia presentaron una menor actividad enzimática de la lipasa hepática en comparación a los controles. No se encontraron diferencias estadísticamente significa- 
Tabla 3. Actividad enzimática de CET P; LCAT; LPL y LH en individuos con hipo e hiper alfalipoproteinemia

\begin{tabular}{|lcccc|}
\hline Actividad enzimática & \multicolumn{2}{c|}{$\begin{array}{c}\text { Hiperalfalipoproteinemia } \\
\text { Casos } \\
(\mathrm{n}=12)\end{array}$} & $\begin{array}{c}\text { Controles } \\
(\mathrm{n}=12)\end{array}$ & \multicolumn{2}{c|}{$\begin{array}{c}\text { Hipoalfalipoproteinemia } \\
\text { Casos } \\
(\mathrm{n}=12)\end{array}$} & $\begin{array}{c}\text { Controles } \\
(\mathrm{n}=12)\end{array}$ \\
\hline CETP $(\% \mathrm{actividad} / \mathrm{hr})$ & $17,0 \pm 2,3$ & $17,3 \pm 2,4$ & $14,7 \pm 3,7$ & $17,4 \pm 4,0$ \\
LCAT (nmol/hr/ml) & $30,7 \pm 1,9$ & $28,3 \pm 1,2$ & $18,6 \pm 1,9$ & $26,2 \pm 1,6^{* *}$ \\
LPL (mmol/hr/lt) & $14,1 \pm 0,5$ & $14,0 \pm 0,5$ & $14,3 \pm 0,7$ & $13,3 \pm 0,7$ \\
LH $(\mathrm{mmol} / \mathrm{hr} / \mathrm{lt})$ & $23,7 \pm 0,8$ & $32,4 \pm 1,8^{*}$ & $40,7 \pm 2,6$ & $36,8 \pm 2,3$ \\
\hline
\end{tabular}

Valores son expresados como promedio \pm ES.

${ }^{*} p<0,05$ versus hiperalfalipoproteinemia; ${ }^{* *} p<0,05$ hipoalfalipoproteinemia.

CETP=enzima de transferencia de ésteres de colesterol; LCAT: enzima esterificadora de colesterol; LPL: lipasa lipoproteica endotelial; LH= lipasa hepática.

tivas con el grupo control en actividad de CETP, LCAT y LPL. En el grupo con hipoalfalipoproteinemia se detectó una significativa menor actividad de LCAT en comparación a los controles. No se detectaron diferencias significativas en la actividad de CETP, LPL y LH.

\section{DISCUSIÓN}

Desde hace varios años se ha establecido la importancia del colesterol-HDL en el desarrollo de la enfermedad cardiovascular ateroesclerótica, la principal causa de mortalidad en la población adulta chilena ${ }^{12}$. Diversos estudios han demostrado que un bajo nivel plasmático de colesterolHDL aumenta en forma independiente el riesgo de ateroesclerosis. Por el contrario, un alto nivel de colesterol HDL es un factor protector al desarrollo de la patología cardiovascular isquémi$\mathrm{ca}^{1}$. En la población adulta chilena, aproximadamente $10 \%$ de las mujeres y $20 \%$ de los hombres presentan bajos niveles plasmáticos de colesterol HDL $^{8}$. Sin embargo, hasta la fecha no se disponía de estudios que evaluaran los mecanismos fisiopatológicos de las alteraciones en los niveles de colesterol-HDL en sujetos chilenos.

Los resultados de este trabajo evidencian que individuos chilenos con hiper e hipo alfalipoproteinemia presentan alteraciones metabólicas específicas que, potencialmente, explicarían los cambios observados en los niveles plasmáticos de colesterol-HDL. En el caso de los sujetos con hipoalfalipoproteinemia, la principal alteración detectada fue una menor actividad de LCAT, en concomitancia con menores niveles de la subfracción de HDL3 y de Apo A-I. La importancia de este hallazgo radica en que estas alteraciones metabólicas pueden estar condicionando una disfunción del sistema de transporte reverso de colesterol $^{13}$, ya que una disminución en el proceso de esterificación de colesterol en la partícula de HDL, genera a su vez una reducción en la capacidad aceptora de colesterol por esta partícula a partir de los tejidos periféricos. Por otra parte, la misma reducción de la fracción de HDL3, correspondiente a las partículas de HDL con mayor eficiencia en la captación de colesterol desde las células periféricas, produciría también una alteración de este proceso, que afectaría en último término el transporte reverso de colesterol. Si bien la reducción de los niveles plasmáticos de Apo A-I observada en los sujetos hipoalfalipoproteinémicos se podría atribuir a una disminución de la síntesis de las partículas precursoras de HDL en el hígado y en el intestino, la explicación más probable está relacionada a un mayor catabolismo de las partículas de HDL que no logran madurar adecuadamente hacia partículas de HDL2, como consecuencia de la menor actividad enzimática de LCAT. Nuestros hallazgos son concordantes con datos previos de pacientes con deficiencia genética de LCAT, los cuales presentan niveles muy bajos de colesterol HDL total, reducción de Apo A-I, de las subfracciones HDL2 y HDL3 y partículas de HDL pequeñas ${ }^{14}$. Desde el punto de vista 
cardiovascular, se ha descrito que estos pacientes presentan un mayor riesgo de desarrollar enfermedad ateroesclerótica clínica, principalmente de tipo coronario ${ }^{15}$.

En los sujetos con hiperalfalipoproteinemia de nuestro estudio, la principal alteración metabólica detectada fue una menor actividad de la enzima lipasa hepática, asociada a un aumento de la subfracción HDL2 y Apo A-I y un mayor porcentaje de partículas de HDL de mayor tamaño en comparación al grupo control. La menor actividad de la lipasa hepática observada en estos individuos hiperalfalipoproteinémicos, produciría una reducción en el catabolismo terminal de la partícula de HDL, con el consecuente incremento de HDL2, la cual es la subfracción de HDL que participa en la fase final del transporte reverso de colesterol a nivel hepático. Adicionalmente, estos pacientes presentan partículas de HDL de mayor tamaño, probablemente atribuible a una falla en el metabolismo selectivo del colesterol-HDL, proceso que es modulado por la actividad de la lipasa hepática7. La situación inversa, pero concordante a nuestros hallazgos, corresponde a las alteraciones lipídicas descritas en poblaciones de Turquía, las cuales presentan un incremento de la masa y la actividad de la enzima lipasa hepática, con una consecuente reducción plasmática del colesterol-HDL total (10 a $15 \mathrm{mg} /$ dl inferiores en comparación a población americana y de Europa occidental), pero principalmente con disminución de la subfracción HDL2 ${ }^{16}$. También nuestros resultados son concordantes con las alteraciones descritas en pacientes con deficiencia genética de esta enzima, los cuales presentan una elevación marcada del colesterol-HDL total, con aumento de ambas subfracciones, pero principalmente del colesterol HDL2, aumento de triglicéridos en la partícula de HDL y predominio de partículas grandes en el plasma ${ }^{17,18}$. Es interesante mencionar que estos pacientes presentan un mayor riesgo cardiovascular, a pesar de tener niveles de colesterol HDL en rangos considerado como «cardioprotectores». Resultados similares se han descrito en estudios de sujetos portadores del polimorfismo $514 \mathrm{C} / \mathrm{T}$ del promotor del gen de la lipasa hepática, en el cual ocurre una sustitu- ción de cisteína a timidina en posición 514 , lo cual se asocia a reducción de la actividad de esta enzima con incremento del colesterol-HDL total, principalmente a expensas de HDL2, aumento de Apo A-I y un mayor riesgo de enfermedad ateroesclerótica prematura ${ }^{19,20}$. De acuerdo a estos antecedentes, nuestros resultados pueden tener interpretaciones aparentemente paradojales. Por un lado, los altos niveles de colesterol-HDL de estos sujetos conferirían un menor riesgo cardiovascular, dada la clásica relación inversa entre prevalencia de ateroesclerosis y niveles plasmáticos de HDL. Por otra parte, existirían subgrupos de sujetos con hiperalfalipoproteinemia que no necesariamente estarían protegidos frente al desarrollo de patología vascular isquémica. Cuando el incremento del colesterol-HDL plasmático ocurre a expensas de una alteración de su catabolismo terminal hepático, se afectaría desfavorablemente el transporte reverso desde la periferia $y$, consecuentemente, podría existir un estado proaterogénico. Es así como se ha reportado que sujetos con una elevación moderada del colesterol-HDL, secundaria a menor actividad de CETP, tienen una mayor prevalencia de enfermedad coronaria isquémica ${ }^{21}$. En nuestro estudio, el seguimiento clínico a largo plazo de los sujetos hiperalfalipoproteinémicos con menor actividad de lipasa hepática en relación a un grupo control con HDL alta y lipasa hepática normal, debiera permitirnos establecer la relevancia pro 0 anti aterogénica del fenotipo metabólico de estos individuos.

Debemos destacar que, si bien dentro del grupo general de sujetos con hiperalfalipoproteinemia la alteración predominante fue una menor actividad de la lipasa hepática, en el análisis individual se detectaron 2 sujetos que presentaban una muy baja actividad de CETP. Este hallazgo nos hace plantear que en estos casos particulares el incremento de colesterol-HDL sería consecuencia de una menor transferencia de colesterol desde las partículas de HDL hacia VLDL, lo cual también podría afectar negativamente el transporte reverso de colesterol hacia el hígado mediado por las partículas de VLDL, y por otra parte disminuiría la capacidad aceptora de colesterol de las partículas de HDL a nivel periférico. También puede especu- 
larse que una deficiencia en la expresión o actividad funcional del receptor de HDL SR-BI, podrían explicar algunos de los casos de hiperalfalipoproteinemia con partículas de HDL de mayor tamaño en que no se detectó cambios en las actividades enzimáticas de lipasa hepática o CETP.

El principal problema de nuestro estudio obedece principalmente a la muestra estudiada, ya que fueron sujetos seleccionados a partir de individuos que acudieron a consulta por dislipidemias 0 chequeo médico. Esto podría constituir un sesgo en el estudio y los sujetos analizados no serían representativos de la población chilena. Además, el

\section{REFERENCIAS}

1. Barter PJ, Rye KA. High density lipoproteins and coronary heart disease. Atherosclerosis 1996; 121: 1-12.

2. Castem WP, Anderson K, Wilson PW, Levy D. Lipids and risk of coronary heart disease. The Framingham Study. Ann Epidemiol 1992; 2: 23-8.

3. Robins S, Cowns D, Wittes J, Papademetriou V, DeEDWANIA P, Schaefer E et al. Relation of gemfibrozil treatment and lipid levels with major coronary events. VA-HIT: a randomized controlled trial. JAMA 2001; 285: 1585-91.

4. Kwiterovich PO JR. The antiatherogenic role of high-density lipoprotein cholesterol. Am J Cardiol 1998; 82: 13Q-21Q.

5. EISENBERG S. High density lipoprotein metabolism. J Lipid Res 1984; 25: 1017-58.

6. TALL AR. Plasma high density lipoproteins. Metabolism and relationship to atherogenesis. J Clin Invest 1990; 86: 379-84.

7. Alvarez V, Cuevas A, Rigotti A. Estructura y metabolismo de las lipoproteínas de alta densidad (HDL). Clínica e Investigación en Ateroesclerosis 2001; 13: 2-8.

8. Berríos X, Jadue L, Pierotic M. Perfil lipídico en la población adulta de la Región Metropolitana. Rev Méd Chile 1992; 120: 331-3.

9. Atger V, Wirbel E, Roche D, Apfelbaum M, Burstein M, GiRard-Globa A. Distribution of HDL 2 y HDL 3 in a random population of healthy French males and females, evaluation by a two step número de casos estudiados es pequeño. Por lo tanto, los posibles estudios futuros en este campo, en nuestro país, deben ser realizados en una mayor cantidad de sujetos más representativos de la población chilena general. No obstante, este trabajo representa, en nuestro conocimiento, el primer estudio que ha evaluado las posibles alteraciones fisiopatológicas del metabolismo del colesterolHDL en sujetos chilenos. Estudios adicionales serán necesarios para establecer la prevalencia real de estas alteraciones en nuestra población, así como también, determinar el impacto de éstas en el desarrollo de la enfermedad ateroesclerótica.

precipitation procedure. Clinica Chimica Acta 1990; 189: 111-22.

10. NiLson-EhLe P, Ekman R. Rapid, simple and specific assays for lipoprotein lipase and hepatic lipase. Artery 1977; 3: 194-209.

11. Manabe M, Abe T, Nozawa M, Maki A, Hirata M, ITAKURA $H$. New substrate for determination of serum lecithin: cholesterol acyltransferase. J Lipid Res 1987; 28: 1206-15.

12. Situación de la Salud en Chile. Ministerio de Salud. Capítulo II: Situación y tendencias 1999; 14-29.

13. TAL AR. An overview of reverse cholesterol transport. Eur Heart J 1998 Suppl A: A31-5.

14. Rader DJ, IKewaki $K$, Duverger $N$, Schmidt $H$, PRitchaRd H, Frohuch J et al. Markedly accelerated catabolism of apolipoprotein A-II (Apo A-II) and high density lipoprotein containing Apo A-II in classic lecithin: cholesterol acyltransferase deficiency and fish-eye disease. J Clin Invest 1994; 93: 321-30.

15. Rosset J, Wang J, Wolfe BM, Dolphin PJ, Hegele RA. Lecithin: cholesterol acyl transferase G30S: association with atherosclerosis, hypoalphalipoproteinemia and reduced in vivo enzyme activity. Clin Biochem 2001; 34: 381-6.

16. Mahley R, Pépin J, Palaoglu K, Maloy M, Kane J, BERSOT T. Low levels of high density lipoproteins in Turks, a population with elevated hepatic lipase: high density lipoprotein characterization and gender specific effects of apolipoprotein $\mathrm{E}$ genotype. J Lipid Res 2000; 41: 1290-301. 
17. Cohen J, Vega G, Grundy SM. Hepatic lipase: new insights from genetic and metabolic studies. Curr Opin Lipidol 1999; 10: 259-67.

18. Conneliy PW, Hegele RA. Hepatic lipase deficiency. Crit Rev Clin Lab Sci 1998; 35: 547-72.

19. Couture P, Otvos JD, Cupples LA, Lahoz C, Wilson PW, SchaeFer EJ, Ordovas JM. Association of the C514T polymorphism in the hepatic lipase gene with variations in lipoprotein subclass profiles: The Framingham Offspring Study. Arterioscler Thromb Vasc Biol 2000; 20: 815-22.
20. Ji J, Herbison CE, Mamotte CD, Burke V, Taylor RR, vAN BOCKXMEER FM. Hepatic lipase gene 514C/T polymorphism and premature coronary heart disease. J Cardiovasc Risk 2002; 9: 105-13.

21. Zhong S, Sharp D, Grove J, Bruce C, Yano K, Curb $D$ ET AL. Increased coronary heart disease in Japanese-American men with mutation in the cholesterol ester transfer protein gene despite increased HDL levels. J Clin Invest 1996; 97: 291723. 